Article

\title{
Designing a Web Spatial Decision Support System Based on Analytic Network Process to Locate a Freight Lorry Parking
}

\author{
Alessandro Crimi ${ }^{1, *}$, Tom Jones ${ }^{2}$ and Antonino Sgalambro ${ }^{1,3} \mathbb{D}$ \\ 1 Istituto per le Applicazioni del Calcolo Mauro Picone, CNR, via dei Taurini 19, 00185 Roma, Italy \\ 2 City of Bradford Metropolitan District Council, Britannia House, Bradford BD1 1HX, UK; \\ tibolticus@gmail.com \\ 3 Management School, University of Sheffield, Conduit Road, Sheffield S10 1FL, UK; \\ a.sgalambro@sheffield.ac.uk \\ * Correspondence: crimi.alessandro@gmail.com
}

Received: 13 August 2019; Accepted: 2 October 2019; Published: 12 October 2019

\begin{abstract}
The relevant role of freight lorry parking facilities as a tool to reduce nuisances and impact of economic activities in densely populated urban areas is widely recognised in the literature. Nevertheless, the literature currently lacks specific contributions addressing the use of a complex Multiple Criteria Decision Analysis (MCDA) approach for coping with an optimal location of freight lorry parking facilities in the urban context. This paper contributes to filling this gap by analysing a real-world case study motivated by the problem of intense freight vehicles traffic around the city of Bradford, Yorkshire (UK). Since it is necessary to include diverse analysis perspectives, reflecting the different classes of involved stakeholders, this study proposes adopting the Analytic Network Process (ANP) approach as a tool to support the selection and evaluation of alternatives for a freight lorry parking facility, followed by the design of software based on this approach. The proposed web Spatial Decision Support System provides a valuable tool to foster extended discussions with experts and facilitate the decision process in this class of location problems.
\end{abstract}

Keywords: multi-criteria decision analysis; spatial decision support systems; analytic network process; locational analysis; stakeholder engagement

\section{Introduction: On the Freight Lorry Parking Location Problem}

The relevant role of freight lorry parking facilities as a tool to reduce nuisances and impact of economic activities in densely populated urban areas is widely recognised in the literature, with many interesting contributions addressing the related decision-making and management issues. For example, in [1] a revealing comparison of the parking practices and problems of freight-vehicle drivers in Gothenburg and Delhi is presented. The study considers parking practices of freight lorry drivers and their opinions about parking availability, using comparative descriptive method and data from official documents and surveys. The study concludes that freight parking problems are strictly related to local factors, therefore it is mandatory to consider each case separately. Moreover, the authors highlight that analysis of freight lorry parking problems cannot be based on car parking models as freight lorry drivers behaviour and issues are rather different from car drivers and introduce different issues.

Another study presents a large and complex survey [2] to define a methodology to analyse how to improve security inside freight lorry parking areas. To this end, the authors evaluate the need for parking slots for freight vehicles by comparing current and anticipated parking slots provision with parking slots demand, calculated using the model developed in [3], and calibrated on the characteristics of the studied area. Security being among the many issues stressed in the survey, the paper compares 
different policy options in relation to EU standards and the associated financial costs. Based on their comparison of the drivers' willingness to pay for a high level of security with the financial costs to achieve it, the authors conclude that such high security parking areas are unlikely to generate any profit.

In [4] a more complex approach is proposed to understand how urban areas would respond to a new parking policy. For this purpose, the authors develop a freight transport traffic simulation. They then apply this simulation tool to model a small study area with two simple policy scenarios. To measure the differences in these scenarios, different elements such as time to find parking and walking distance to the final destination are calculated. The results of this study provide a fairly accurate description of the consequences of different policy scenarios; however, given that this kind of approach apparently requires a deep understanding of local freight vehicle flows, it would be hard to apply over a large area.

Although the above mentioned articles provide useful insight on freight transport logistics problems and guidelines on the elements and classes of data that mainly affect the public's decisionmaking on freight transport logistics, none of these articles addresses the need for a practical decision support tool for location analysis purposes. Many examples of Spatial Decision Support Systems (SDSS) proposed in the literature have integrated simple Multiple Criteria Decision Analysis (MCDA) methods with the use of data visualisation techniques to enhance understanding of the spatial characteristics of location decision problems [5], but only a few address the problem of locate parking facilities through participative decision making processes.

Among these, Jelokhani-Niaraki and Malczewski propose in [6] an integration between Geographical Information Systems (GIS) and MCDA to select the most suitable sites for public parking facilities in the capital of Iran, Tehran. Their approach is aimed at supporting a collaborative spatial planning process and is based on two decision rules. The first rule is based on an Ordered Weighted Averaging-based approach for generating individual solutions and is devoted to define the individual ranking of alternatives. The second rule exploits a Borda-based method to aggregate the individual solutions into a collective consensus solution. An interesting discussion on the limitations for user-centred participative policy making processes is included in the paper. A tool for locating public parking facilities in Tehran is also considered in [7], where the collaborative decision making process is developed through the use of geo-social networks. The Telegram application interface is adopted here as a client-side GUI. Their approach builds on a sequential scheme: selection of dominant criteria, adoption of AHP as a MCDA tool for weighting alternatives, fuzzy majority approach to aggregate opinions of the different levels of decision-makers, and final selection of the best alternative. The method turns out to be rather independent from the considered application, hence rather versatile in principle, but somewhat generic as well, in that some peculiar features of the considered public policy making problem might be not fully captured in its conceptual design. In [8] a multi-method approach based on stakeholders analysis, cognitive mapping and Multi-Attribute Value Theory is proposed as an integrated MCDA tool for approaching policy problems, and applied on a case study to support the evaluation of alternative public parking areas in the touristic region of Alberobello (Puglia, Italy). According to the authors, limitations of the above mentioned approach are mainly related to a not easy inclusion of divergent views during the weight elicitation process. The same case study is considered in [9] for what concerns the design of alternatives, focusing on how to expand the solution space and discover new alternatives for a public policy making problem.

To the best of our knowledge, the current literature lacks specific contributions addressing the use of a complex MCDA approach for coping with an optimal location of freight lorry parking facilities in the urban context, a territorial policy problem which presents many specific features, including the characteristic of lorry parking being often considered as a non-desirable (obnoxious) facility by resident citizens. Therefore, this paper intends to contribute to filling this gap, while analysing a real-world case study motivated by the problem of intense freight vehicles traffic around the city of Bradford, Yorkshire (UK). Since it is necessary to include diverse analysis perspectives, reflecting the different 
classes of involved stakeholders, this study proposes to adopt the Analytic Network Process (ANP) as an approach to support the selection and evaluation of alternatives for a freight lorry parking facility, complemented by the complete architecture design of a wed Spatial Decision Support System.

The remainder of this paper is organised as follows. Section 2 provides a description of the real-world freight parking problem in the City of Bradford, followed by more detailed presentation of the research objectives of this paper. Section 3 briefly outlines the steps and main advantages of the ANP method in comparison with similar methods, and then sets out in detail the application of ANP to the problem described in Section 2. Section 4 provides some assessment of the advantages of building a web Spatial Decision Support System and guidelines on the architecture of such software. Finally, Section 5 analyses the impact and the main findings of the performed study and highlights areas for further development.

\section{Problem Description and Research Objectives}

Bradford is the UK's fifth largest city, with 522,452 citizens living within the city boundaries, and has the youngest and fastest growing population of any city outside London [10]. The main connection of this large city with the national motorway network is via the M606, a spur of the M62 motorway which is located south of Bradford. Considering these factors, it is not surprising that the road network to the south of Bradford is affected by intense freight vehicles traffic. In fact, in 2016 the M606 was estimated to have an average daily flow of 5644 freight vehicles [11]. The flow is illustrated in map Figure 1, centring on the M606 with the M62 on the bottom right, with a green to red scale used to indicate the intensity of daily freight transport on the main roads.

The Driver Hours Regulations is the principal set of laws governing day to day working time and break/rest period requirements in the EU, imposing mandatory breaks and rest periods of different extent for freight vehicle drivers. Given the inherent nature of driving work, it is generally not possible for drivers of freight vehicles to take break periods at their home depots and they therefore need to park their vehicles while these break periods are undertaken. That implies a demand for suitable lorry parking facilities where vehicles can be safely parked. Given the lack of such facilities in the Bradford area, drivers are likely to park inappropriately on the public highway shoulder or other unsuitable locations.

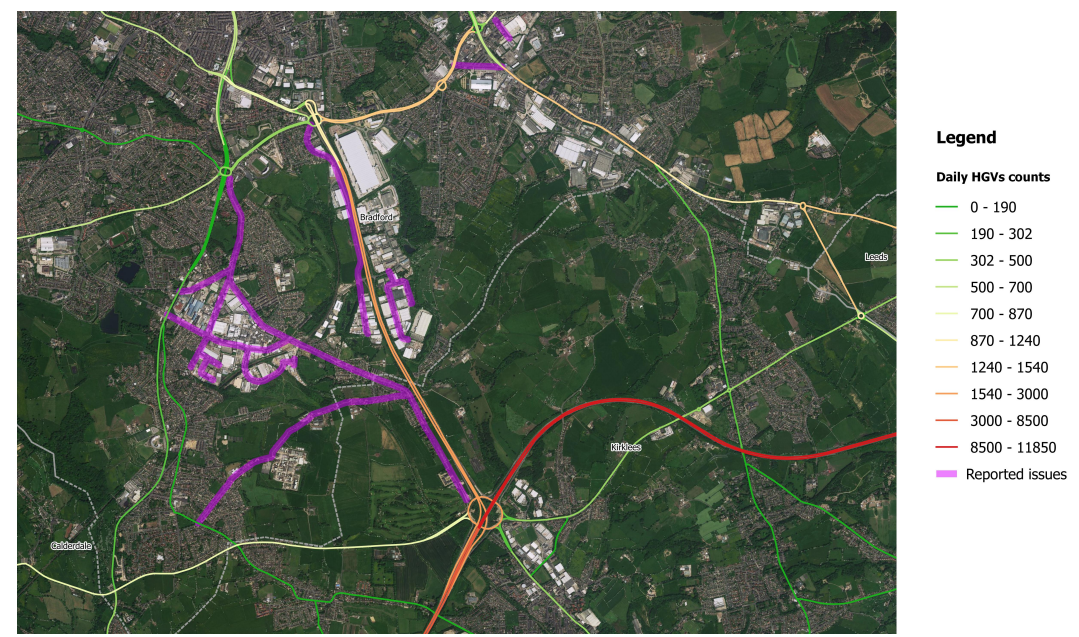

Figure 1. South Bradford.

In the last decade, the southern part of the Bradford district has been subject to sporadic but constant issues related to inappropriate parking by heavy goods vehicles (HGVs). The areas of interest are represented in violet in map Figure 1. The observed consequences include:

- Traffic flow interference and congestion

- Depositing of various types of litter by HGV drivers 
- Theft of goods from curtainsider trailers

- Noise pollution (running engines, refrigeration units) emitted by lorries

- Damage to pavement or footpath infrastructure

- Visual intrusion of lorries

Such issues could be tackled by establishing a dedicated lorry parking facility, which in turn would require the preliminary selection of a range of possible candidate sites, from which to identify the optimal location in the area.

\subsection{Purposes of This Research}

Locating a lorry parking facility, although highly useful in reducing nuisances arising from freight transport, can be considered an obnoxious facility location problem (see for instance [12]), since such a facility is likely to attract an increased HGV traffic in the interested areas and often draws social opposition from resident citizens. Moreover, the process of locating a lorry parking facility requires collection and elaboration of a large quantity of social, economic and environmental data. For these reasons, the location of a lorry parking can be considered as an inherently complex strategic decision-making problem, as it involves different interconnected elements and must achieve objectives that are often in conflict. The above described strategic decision making problem falls into the class of semi-structured decision problems and presents a substantial involvement of subjective judgements from different classes of stakeholders (see also [13]). Accordingly, MCDA appears a more suitable model component for building a Spatial Decision Support System with respect to purely Optimisation-based approaches (including mathematical programming and meta-heuristics) given the absence of a complete set of reliable and objective parameter to feed an explicit mathematical formulation of objective functions and constraints. As also apparent from the state-of-art in Section 1, AHP is often adopted for coping with public policy making problems in the context of companies and organisations. Nevertheless, it presents some renown limitations that might prevent its application in lack of given assumptions (see [14] for a detailed list of the AHP method axioms), posing the need to identify a suitable method that might be applicable in the specific case under investigation.

In order to allow for an appropriate involvement of the stakeholders while collecting data on preferences and a valuable support of the decision maker throughout the interactive and iterative process, the design of a Spatial Decision Support System needs to embed ad-hoc components, often referred to as Stakeholder Components [5]. Note that an efficient involvement of stakeholders is nowadays facilitated by the availability of web-based tools to foster seamless participation, interaction and expression of preferences by end-users, experts and decision makers, emphasising the relevance of an appropriate choice of the technologies to be adopted.

In this paper, we aim therefore at addressing the following research objectives:

RO.1 Identify the classes of stakeholders involved in a lorry parking location problem and their respective objectives (stakeholder analysis);

RO.2 Evaluate ANP as an MCDA approach to be adopted as an underlying model component for the considered Spatial Decision Support System;

RO.3 Study the set of elements to be included in the SDSS to take into account all the factors influencing the decision making process;

RO.4 Conceptualise and represent the dependency relationships among the considered elements (dependency network modelling);

RO.5 Devise the architecture of the proposed Spatial Decision Support System;

RO.6 Beyond the conceptual design: identify the most suitable technological tools to allow for a practical and successful implementation of the proposed SDSS. 


\section{Analytic Network Process Approach}

In this paper we consider the use of a multiple criteria decision analysis method to rank all the locations potentially suitable for siting a freight lorry parking.

In line with objective RO.1, the first subsection deals with the analysis of the stakeholders and their objectives. The main task here is to identify the relationships between different classes of stakeholders and the issues they care about most, as these will constitute the basic elements in developing our Spatial Decision Support System. Following such analyses, and consistently with research objective R0.2, we explore the possible use of ANP as a suitable model component for our SDSS. Although we first also considered using the Analytic Hierarchy Process (AHP) method, we soon realised that the complexity of the problem rendered this method unsuitable. The characteristics of this problem cannot in fact be hierarchically represented, as it involves logical interconnections between elements in a complicated dependency structure that allows elements on different levels to influence each other. Moreover, a purely hierarchical model cannot encompass the case of alternatives potentially influencing the weights associated to the criteria. Since in the lorry parking location problem the elements influencing the decision-making process turn out to be highly interconnected, the Analytic Network Process (ANP) permits to model the problem in a more complete way by controlling and estimating all the element interconnections. That is possible because ANP is able to generalise the AHP approach by allowing the presence of any type of possible mutual influences among the elements [15]. In the ANP method, the problem is structured as a network, and its elements are evaluated through a pairwise comparisons approach to measure their weights in the model, which, after some matrix calculations, allows ranking of the alternatives considered in the problem. A detailed description of the proposed ANP method is provided in Section 3.2, where we focus on the process of constructing the ANP network model for the specific lorry parking location problem. To this aim, we first outline the main phases of the ANP (RO.2) and then construct the ANP dependency network model based on the outcomes of the stakeholder analysis (RO.3-RO.4).

\subsection{Stakeholder Analysis}

In the process of making a decision on a public matter, the starting point for the theoretical model should be the various stakeholders and their interests. The stakeholders are those individuals or organisations that have personal interest and influence in the decision process because they have goals directly or indirectly affected by the problem and its possible resolution [16]. Consequently, the first essential step in a public decision-making problem process is classification of the stakeholders involved and their interests. The stakeholders identified in our decision-making problem alongside their main objectives are drafted in Table 1.

Table 1. Involved stakeholders and their objectives/resources.

\begin{tabular}{lll}
\hline Stakeholders & Main Objectives & Resources \\
\hline Local residents & maximise distance from residential areas & cognitive/political \\
Freight vehicle drivers & minimise deviation from planned routes & economic \\
City Council & minimise nuisances & political/economic \\
Local business & maximise delivery security & cognitive \\
Freight lorry parking owners & maximise profitability & economic \\
\hline
\end{tabular}

As expected in a real problem scenario, we can see that there are multiple stakeholders with different and conflicting interests. Local residents are directly affected by inappropriate HGV parking, as highlighted in Section 2, and their main interest is to maximise the distance between their homes and HGV traffic and hence the freight lorry parking. Local residents are the primary source of information on inappropriate parking through their reporting of nuisances to local authorities, and these complaints are the spur for political action to tackle the problem. Local businesses are also affected by inappropriate parking, as road congestion causes delay in incoming deliveries. Because local businesses are the main 
factor in the generation of HGV flow, through their involvement it is possible to obtain information about the HGV flow that would otherwise be difficult to obtain, such as the number of daily deliveries in the area. City Council holds an indirect interest in the problem since it is concerned with the general well being of the local population; hence, it could decide to provide financial support for construction of a freight lorry parking and enact policies to discourage inappropriate parking. To ensure a positive revenue stream, a freight lorry parking needs to be attractive to drivers, which means it should offer security for their goods and access to amenities while avoiding costly HGV deviations from their original routes. Freight lorry parking owners can be interested in the project as it represents a long-term financial investment.

\subsection{Building an ANP Model for the Considered Problem}

Let us start by outlining the main phases of the ANP method as a Multiple Criteria Decision Making tool as described in [17]. The ANP is a generalisation of AHP, and is more appropriate for dealing with real decision-making processes with complex dependency structures between the considered elements.

The first phase is aimed at developing the network representing the decision-making process. This task first requires clear definition of the decision objective. Then, all the criteria specifically affecting the decision-making process need to be classified into groups or clusters, each group containing criteria with similar meaning. The last elements to be identified are the alternatives or options from which the best solution can potentially be selected. The relations among the elements (criteria and alternatives) within the network dependency structure are represented by arrows and these relations are the key factors in gaining a more precise representation of a real problem than it would be possible by using a more rigid hierarchical structure. For example, it is possible to identify feedback relations linking the alternatives and the criteria and dependency between criteria of any cluster. Additionally, clusters are correlated to each other through inheriting relations from their elements.

The second phase entails making pairwise comparisons. These can be divided into two main types: comparison between clusters, which provides a general understanding of the problem, and comparison between elements, which is more in depth and specific. This process is needed to determine the relative weight of two elements with respect to another criterion in the network influenced by both elements. These comparisons can be either qualitative or quantitative. In qualitative comparisons, first, two elements are compared by choosing the appropriate verbal assessment from a list to express which one has more weight and how much weight they have in relation to a third criterion; then, the verbal assessment is translated into a number between 1 (when elements have the same weight) and 9 (when one element has much greater weight than the other). In this way it is possible to cope with intangible criteria, since it is not necessary to assign values to the criteria. Meanwhile, in quantitative comparisons, value is assigned to the two alternatives in relation to the criterion being considered, with the larger alternative value considered as a multiple of the smaller one, but bearing in mind that the ratio should always be between 1 and 9 .

Given an element of the network and a cluster, the associated comparison matrix is defined as a matrix where the entries are the numeric values of all the pairs of elements in the cluster influencing the selected element. The cluster comparison matrix is defined in the same way but with the difference that the entries are pairs of clusters. The main eigenvector of these matrices synthesises the judgements determined with the pairwise comparisons.

The third phase is the gradual generation of three matrices. The first to be generated is the unweighted or initial supermatrix, in which the entries are indexed by pairs of elements of the dependency model, and the column associated with an element is made by the main eigenvectors of all its comparison matrices. The cluster comparison matrix eigenvector is used to weight the elements of the corresponding column blocks of the initial supermatrix, resulting in the creation of the second matrix, or weighted matrix, which is a column stochastic matrix. The weighted matrix is then raised to a limiting power, in order to converge into the last matrix, or limit matrix, thereby producing a 
long-term stable set of weights that represents the final priority vector, as stated in the Perron-Frobenius theorem [18].

To identify the elements that influence the location of the parking facility problem we took into account all the information gained during the stakeholder analysis. These elements along with brief descriptions are listed in Table 2.

Table 2. Analytic Network Process (ANP) elements and descriptions.

\begin{tabular}{|c|c|}
\hline Elements & Descriptions \\
\hline Level of use & $\begin{array}{l}\text { Potential use for a new parking areas based on data collected through manual } \\
\text { counting of the highway network flow }\end{array}$ \\
\hline Distance to residential areas & The residential areas are all currently or potentially inhabited areas \\
\hline Running costs & $\begin{array}{l}\text { Preliminary estimate of the annual maintenance costs related with the } \\
\text { built facility }\end{array}$ \\
\hline Profitability & $\begin{array}{l}\text { Preliminary estimate of the revenues associated with the facility and the level } \\
\text { of use }\end{array}$ \\
\hline Building costs & $\begin{array}{l}\text { Preliminary estimate of the construction costs related with the size and } \\
\text { the facilities }\end{array}$ \\
\hline Nuisances covering & The coverage of areas subject to inappropriate HGV parking \\
\hline Size & The potential physical dimensions of the build \\
\hline Land cost & Preliminary estimate of the land purchase costs in relation to the land size \\
\hline Land policy & Whether there is a constraint on possible uses of the land \\
\hline Accessibility & $\begin{array}{l}\text { This attribute evaluates how easy it is to reach the location from the main } \\
\text { truck routes }\end{array}$ \\
\hline
\end{tabular}

After the identification of all the elements, the relationship network was constructed, as depicted in the graphical representation in Figure 2.

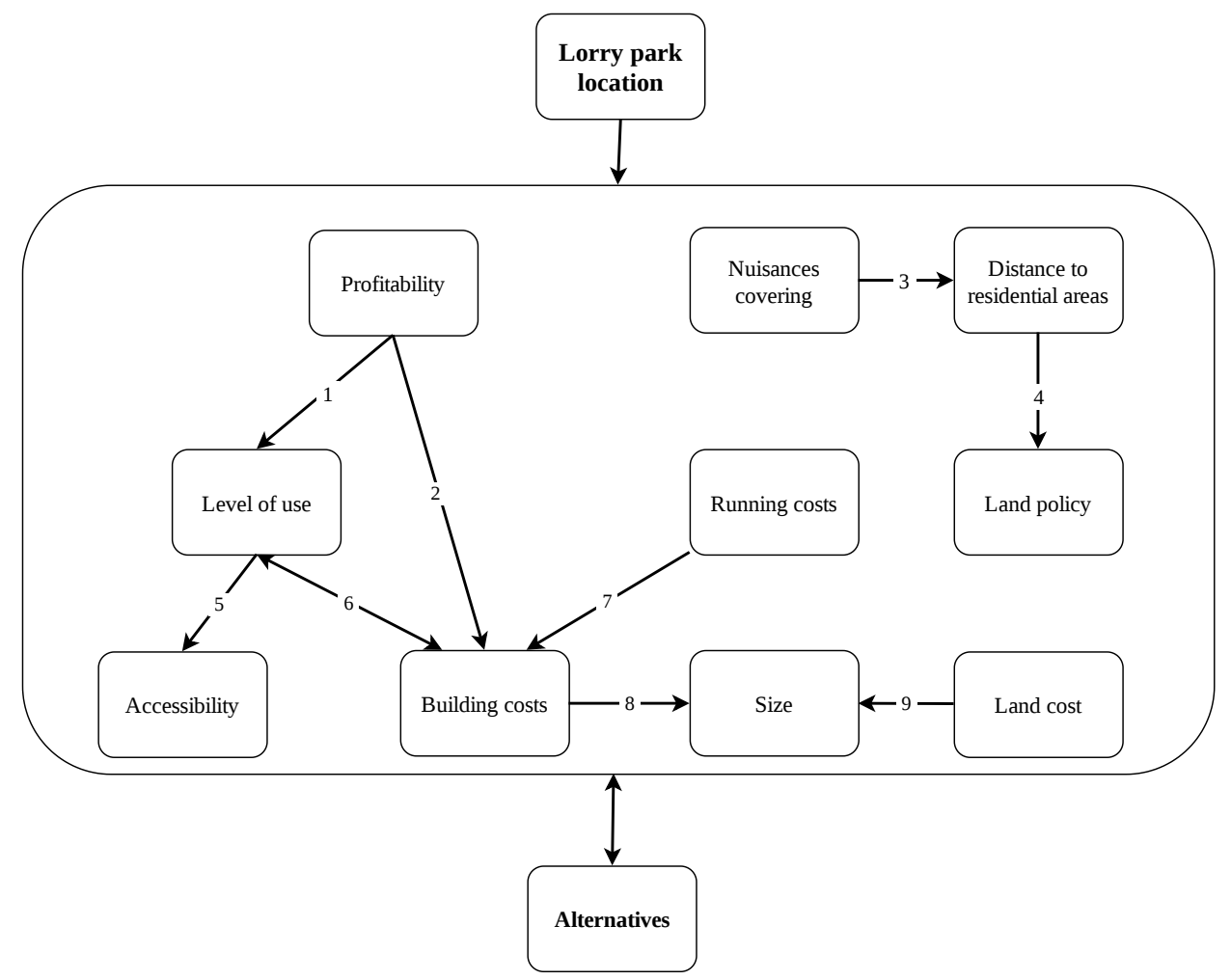

Figure 2. Analytic Network Process dependency graph. 
In Figure 2 the arrows between elements represent the dependency relations, while the arrows that connect the central area with other elements are considered as connected to each element of the delimited area. The set of identified dependency relationships are explained here in more detail, by associating each specific presented concept to the related arrows in the graph depicted in Figure 2 through the use of numerical labels. Profitability is largely influenced by the level of use of the facility: the higher the number of vehicles stopping overnight, the highest the economic sustainability of the overall parking system (1). A good Accessibility of the parking facility fosters its Level of use (5), as the former turns out to be a relevant element influencing lorry drivers when making their decision on whether or not to use a facility. Indeed, a substantial detour with respect to the their original route might be not convenient from the economic point of view, and even cause extended delays which might prevent lorry drivers from utilising the parking facility. Furthermore, in those extreme cases where the facility will not be accessible to heavy good vehicles (this is the case for locations connected by roads not suitable for HGV), the parking facility would not be usable at all by lorry drivers. This is also the reason for Accessibility being considered as a constraint in the clustering of elements depicted in Figure 3. By allowing increased economic investments (Building costs) it would be possible to provide better services to the drivers (including showers, hot meals and filling stations) who would be in turn more interested in the parking facility, securing a growth in the Level of use (6), and could be even willing to pay a higher fee, thus affecting overall the Profitability as well (2). On the other hand, this might lead to increased fixed and variable Running costs, particularly in those periods characterised by a low turn out (7). The Size of the selected location for installing a lorry parking facility affects both the Building Costs (8) and the Land costs (9), which increase as the size of the location grows. A major analysis factor for the choice of the appropriate location is the extent to which the effects of installing a lorry parking facility in a given location are spread over the relevant geographic area (Nuisances covering), thus enabling a sufficient coverage on those spots interested by the social issues already presented and discussed in Section 2 . The latter needs to be counterbalanced with the location being concurrently far enough from the residential areas. Indeed, a facility directly located within the areas affected by the nuisances would be certainly effective in solving those issues strictly related to inappropriate parking by heavy goods vehicles, but at the same time would possibly produce different nuisances affecting residential areas due to increased traffic of those HGV headed to the parking facility (Distance to residential areas). Such a complex spatial relationship is represented by arrow (3), where the influence of the latter element on the former is captured. Finally, arrow (4) is devoted to account for the impact of the Land policy element on the Distance to residential areas one. Indeed, the suitability of a given area as a feasible alternative for siting a lorry parking, whilst respecting all constraints on the distance from residential areas, is affected in turn by the intended use of all the areas close to the candidate location, as in the future these might be allocated for residential development.

Following the ANP method procedure, those elements playing similar roles in the decision making process are grouped to form clusters, and the groups identified in this case and their relations are shown in Figure 3, where an arrow connects two clusters whenever these include elements presenting a mutual influence.

The Benefits cluster includes the elements securing some form of favourable contribution, whereas the Costs cluster is associated with those providing an unfavourable contribution, and Constraints includes those elements essential to some extent for the feasibility of the facility.

The presence of loops in the cluster graph accounts for an influence occurring between different elements included in the same cluster, and confirms the apparent complexity of the dependency network structure for this decision making problem which motivated the choice for an ANP approach. 


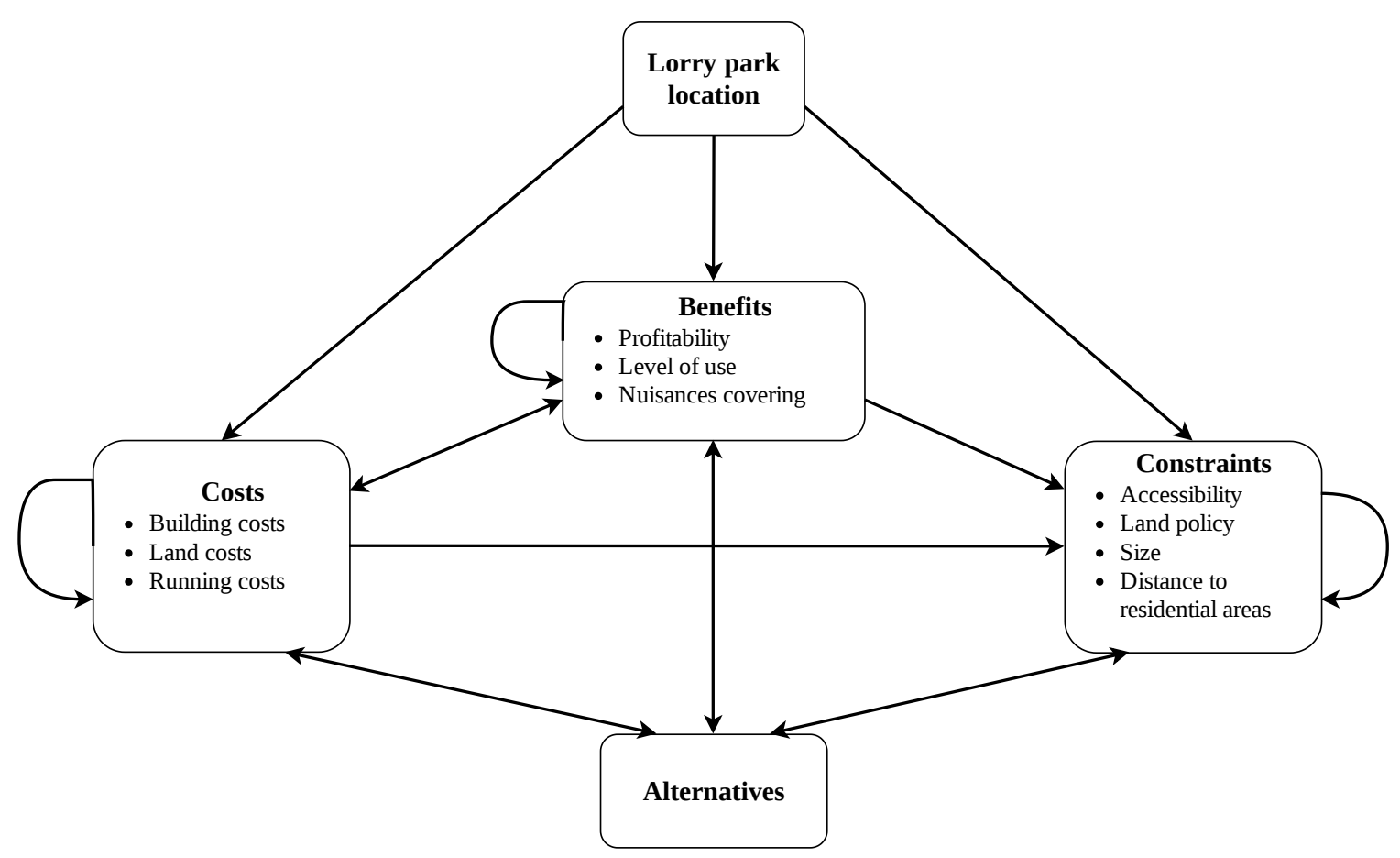

Figure 3. Analytic Network Process cluster graph.

\section{Architecture of the Web Spatial Decision Support System}

A $\mathrm{iO}^{\prime} 00^{\prime \prime}$ (WSDSS) is a web based system designed to assist in the handling and visualisation of geographical data (also called spatial data) relevant to the decision-making process [5]. It presents the potential to deal with a large variety of location problems including those posed by the freight parking location problem. Developing a specialised WSDSS would be an important tool in addressing the above-mentioned issues and would also encourage stakeholders' participation in the decision process. Hence, in this section we discuss a possible architectural design for a specific WSDSS to rank the identified alternatives, using the ANP method described in Section 3.2 as a model component, thus addressing research objective RO.5. To elucidate how the WSDSS should operate, the next subsection gives a comprehensive description of the interaction process between the user and the software. We then conclude the architectural design description by providing tailored specifications for all of the architecture components that are considered appropriate for the proposed WSDSS, by explaining their mutual interactions and including indications on the technologies best suited for developing each component, as per research objective RO.6.

\subsection{WSDSS General Process Description}

The process described in this section can be followed on Figure 4. The first web page provides a map that the user can navigate to select the area of interest for the location problem (Select location). Once the area is selected the software identifies all spatial data sets in that area already present in the software database (spatial query on existing geodata) and enables the user to select spatial data sets relevant to the location problem (display local geodata). If the existing spatial data are not sufficient to model the location problem, users can also add or create their own spatial data set using Geographic Information System (GIS) tools, and new spatial data sets can be stored on the user online library for future use (select/modify/add relevant geodata). All spatial data sets in use can be visualised on the web map, which makes it easier for the user to select an appropriate existing ANP dependency model and modify it to best match the decision problem or to create a completely new model (structure the dependency model). Whenever possible, each criterion in the model should be associated with one of the spatial data sets to minimize the burden of the needed qualitative comparison, thus speeding up 
the pairwise comparison phase. When the user has completed the dependency model the software can then generate the comparisons tables to be filled in (display comparison tables). The comparisons tables and the dependency model can be also shared with other users in order to allow participation of stakeholders and experts in the decision-making process, through inputting their own opinions into the tables (fill comparison tables). Involving stakeholders is particularly important in cases of public decisions, where a single problem can affect a multitude of people, who could have an interest in directly influencing the decision process. Once all the interested persons have had the opportunity to fill in the tables, the software uses a mean to synthesise all the input values and fill in the comparison matrices that will be used for subsequent calculations.

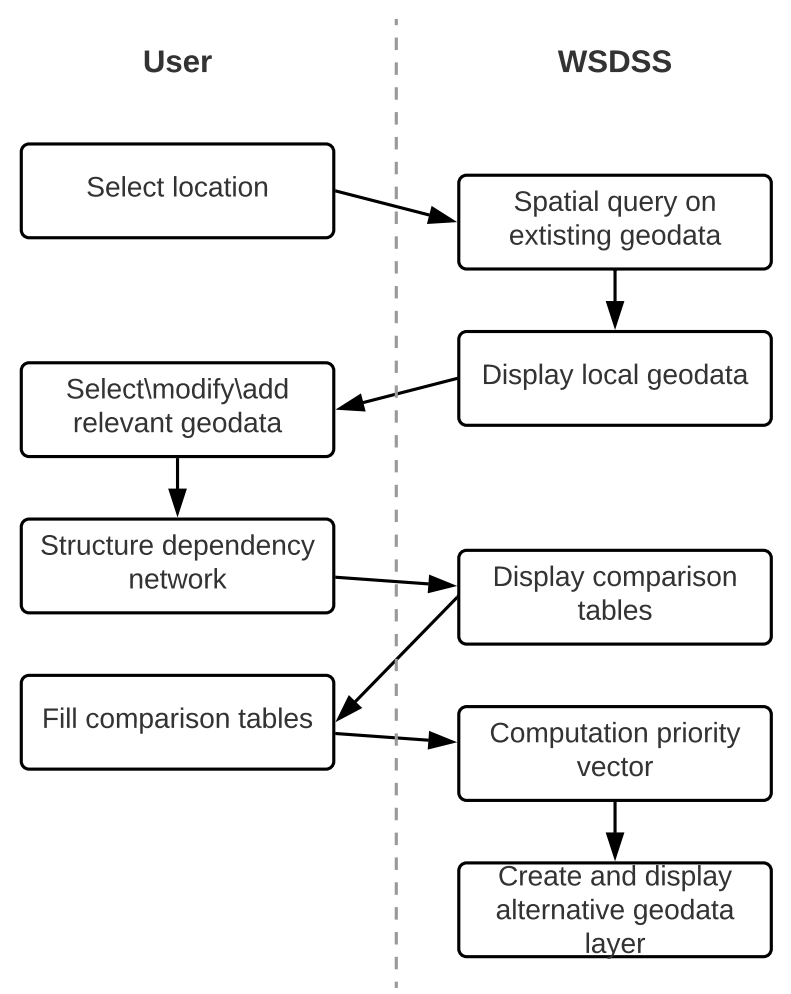

Figure 4. Process chart.

The stakeholders' judgements could also be seen as a survey giving insight on the general perceptions of the problem under consideration. Based on the filled in comparisons tables the software calculates the main output of the software, the priority vector of the alternatives (compute priority vector), which should also be represented as a results layer on the web map, where each alternative is represented based on its final score (create and display alternatives geodata layer). To ensure the overall transparency of the decision-making process, the model and all spatial data sets and tables should be made accessible to the public through on-line sharing.

\subsection{Proposed WSDSS Components and Design}

The proposed WSDSS architecture is schematically represented in Figure 5, while in Table 3 the technology suggested to implement each of the WSDSS components is recalled and briefly motivated. 


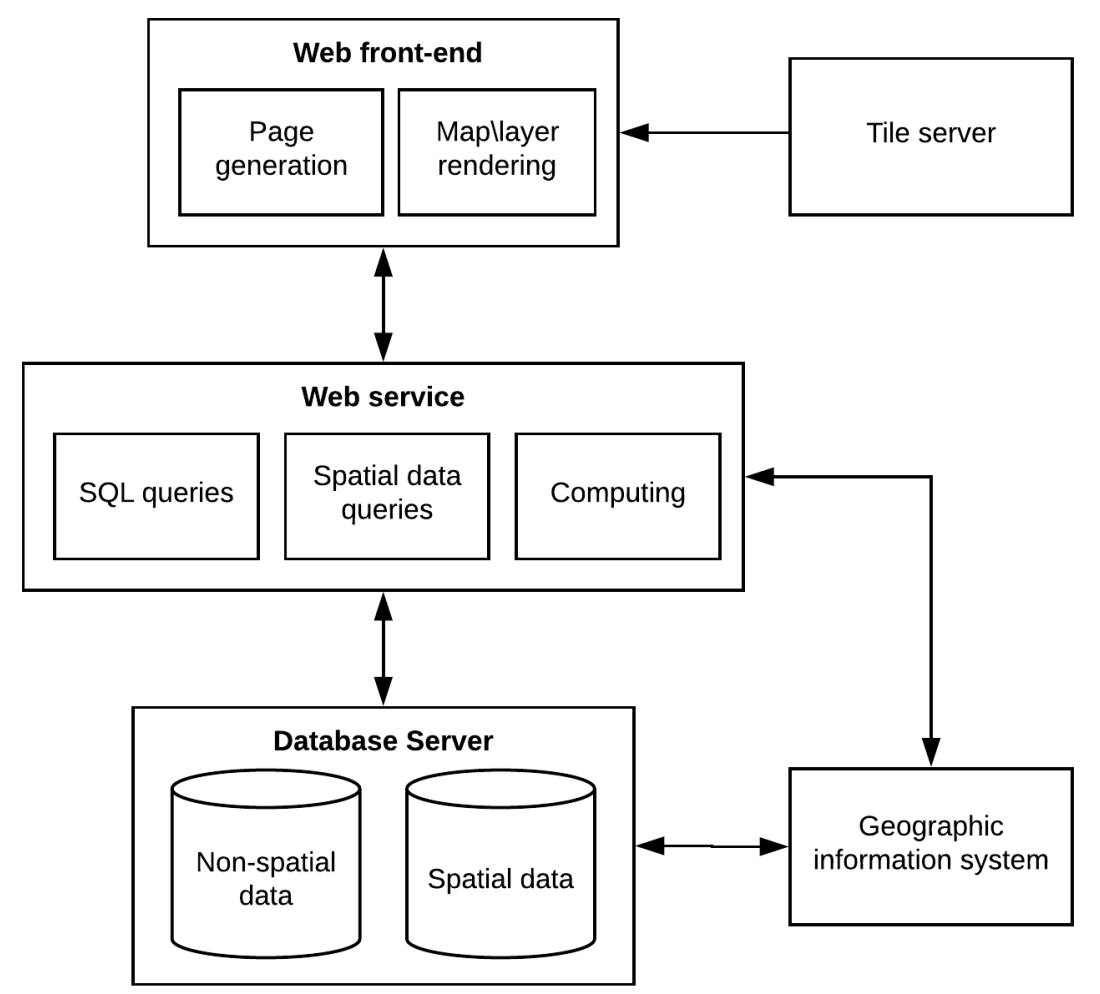

Figure 5. Web Spatial Decision Support System (WSDSS) architecture.

Python is the programming language suggested for the software development due to its flexibility and easy integration with all the components. The front-end of the software oversees the interaction with the user and is responsible for the acquisition of inputs that are then sent to the appropriate component. We also suggest using Flask web framework for the main development of this component (page generation). Flask is written in Python and has a simple, easy to use core, but supports a large variety of extensions capable of doing everything necessary. A popular way to display and navigate maps on-line is via the tiled web map, which is constructed through seamless joining of individual small image files. The tiled map has several advantages over older methods which usually display a single large image; for example, when the user moves around on the map only new tiles are loaded but most of the tiles can be kept displayed, which greatly improves the movement fluidity compared to loading a single map image for the whole view-port. For this kind of map, it is essential to use an existing public tile server or build it with Mapnik, a free tool-kit for developing mapping applications, we chose Mapnik because it allows greater customisation (tile server). Mapnik is written in modern $\mathrm{C}++$ and has Python bindings. Then the map generated by the tile server and data layers coming from the web service component can be downloaded and displayed using Leaflet (map/layer rendering). Leaflet is an open source JavaScript library widely used to build web map applications. All the shaping tools for spatial data layers displayed in the front-end should be connected with tools accessible on QGIS, a geographic information system application that supports viewing, editing, and analysis of spatial data. The data created need to be stored, and PostgreSQL is a classic choice in Python for storing data with fixed structure such as: users account information, past queries, model structures, etc. (non-spatial data). PostgreSQL can be extended with PostGIS to support specific spatial data storage tasks (spatial data), allowing location queries to be run in Structured Query Language (SQL). The last and arguably the most important component is the web service, which can deal with all the logic necessary in this WSDSS. The web service should be written in Python and extended with libraries for each specific task. SQLAlchemy is an open source SQL toolkit that is commonly used to retrieve and query SQL databases (SQL queries), and which needs to be extended with GeoAlchemy 2 to add support for spatial 
data. Once the pairwise comparisons have been submitted by the user, the web service can, by using NumPy library, deal easily with the large matrices generated and compute the final priority vector as described in Section 3.2 (computing). The priority vector for the alternatives is then sent to QGIS to generate spatial data that are displayed on the front-end map as a final result.

Table 3. WSDSS components and suggested technology.

\begin{tabular}{lll}
\hline Component & Technology & Motivation \\
\hline Tile server & Mapnik & customisable \\
GIS & QGIS & open-source, easy integration with Python \\
Page generation & Flask & easy, supports extensions \\
Map/layer rendering & Leaflet & open source, small code footprint \\
SQL queries & SQLAlchemy & efficient, high-performing database access \\
Spatial data queries & GeoAlchemy 2 & extensions for spatial database access \\
Computing & NumPy & supports the calculation of large matrices \\
Database & PostgreSQL & advanced relational database \\
Spatial database & PostGIS & extensions for spatial database \\
\hline
\end{tabular}

\section{Discussion and Conclusions}

In this paper we presented the conceptual and architectural design of a web Spatial Decision Support System adopting the Analytic Network Process as a tool to optimally locate a freight lorry parking within a set of possible candidate alternatives. In this last section we first elaborate on the main findings of our research against each of the research objectives presented in Section 2.1, then limitations and further developments of our work are discussed.

Following research objectives RO.1, we assumed the identification and involvement of all the stakeholders as the first step to enable the use of multiple-criteria decision-making techniques in the considered context. Findings from such a stakeholder analysis turned out to be particularly relevant as they highlighted the presence of several stakeholders with potentially conflicting objectives. Outcomes of the stakeholder analysis were also instrumental for feeding all the next conceptual and architectural WSDSS design phases, by allowing a richer insight in the characterisation of the decision making process. Indeed, as compared with results from related works on application of MCDA to public parking facility location, our application presents some peculiar characteristics already apparent from the stakeholder analysis, which became further visible as an outcome of the dependency network analysis (RO.3 and RO.4), where all the important elements involved in this location problem, and their mutual relations, were analysed in detail. In the clustered graph arising from this process, elements were grouped according to their roles in the decision making process and all inter- and intracluster relationships became visible.

Through the development of research objective RO.2, and in view of the results from the dependency network analysis, we could see how ANP seems an appropriate choice for an MCDA method in this field, due to its suitability for modelling specific problems presenting particularly complex logical interconnections. Recall for instance the presence of loops in the clustered graph: these would have been forbidden in any AHP hierarchy, thus confirming the need for more sophisticated tools such as the Analytic Network Process adopted in our research as a MCDA approach to parking facility location.

It is an open question whether a similar behaviour might be observed in the dependency network structure for different applications, such as public parking location, when developing a deeper stakeholder and element dependency analysis. Indeed, in some of the recent contributions in this field, adopting simpler MCDA approaches and recalled in the state-of-art section of this paper, a preliminary stakeholder analysis was not performed.

The next stage of this research was aimed at presenting the conceptual design for a web Spatial Decision Support System implementing the above described ANP method as a model component (RO.5); first, the process was explained, then a possible architecture was sketched, along with 
suggestions for appropriate technologies for dealing with each component, thus addressing objective RO.6 as well. The results arising from research objective RO.5 in terms of WSDSS design are of particular interest in respect to the impact of the architecture components to secure the design of a participative decision making tool, involving as many stakeholders as possible in the evaluation of alternatives. This turns out to be extremely relevant in our lorry parking location process, based on the potential obnoxious role of a freight lorry parking facility in attracting increased amounts of HGV traffics.

A further interesting outcome of this research is that it reveals the possible use of open source technologies, as detailed in the relevant section, when implementing the identified components for the WSDSS. In view of the involvement of local authorities as potential promoters of the WSDSS adoption, this aspect introduces an apparent advantage in terms of economic costs of the implementation process and transparency of the decision making process.

Limitations of this work can be identified in the WSDSS being extremely tailored on the specific application. Despite its validity can be easily extended for lorry parking location problems in different geographical contexts, it is not straightforward to export the proposed modelling scheme to other fields of territorial policy making, as the conceptual design of its model component is inherently associated with the outcomes of the stakeholder and dependency network analyses detailed in Section 3. Still, such a limitation is largely counterbalanced by the expected valuable social and economic advantages arising from the use of a decision support tool which, stemming from a deeply articulated representation of the specific decision making problem (location of freight lorry parking in urban areas), guarantees an increased applicability and reliability of the research results for practical purposes.

Future developments of this research include technical implementation of the WSDSS, together with feeding and calibration of the model based on the decision-makers' selection of location alternatives and related economic and financial assessment, also including a practical collection and analysis of the outcomes of stakeholders' involvement. At this stage, for confidentiality reasons, it was not possible to collect information on candidate locations and present the outputs of the proposed model. These will be hopefully included in future developments of the present research work.

The WSDSS structure proposed in this paper also represents a valuable tool to foster extended discussions with experts and to design similar tools facilitating the decision process for different classes of location problems.

Author Contributions: All authors actively contributed to the various phases of the research and the writing of the paper. During the development of this research, under the scientific direction of A.S., A.C. has been working as a secondee at the City of Bradford Metropolitan District Council, supervised by T.J., in his role as Senior Transport Planner at the Development Plans \& Transport Planning Department.

Funding: This research was partially supported by the project "Promoting Sustainable Freight Transport in Urban Contexts: Policy and Decision-Making Approaches (ProSFeT)", funded by the H2020-MSCA-RISE-2016 programme (Grant Number: 734909). This support is gratefully acknowledged.

Conflicts of Interest: The authors declare no conflict of interest. The funders had no role in the design and development of this study, in the writing of the manuscript, or in the decision to publish the results.

\section{References}

1. Malik, L.; Sánchez-Díaz, I.; Tiwari, G.; Woxenius, J. Urban freight-parking practices: The cases of Gothenburg (Sweden) and Delhi (India). Res. Transp. Bus. Manag. 2017, 24, 37-48. [CrossRef]

2. Carrese, S.; Sandro, M.; Nigro, M. A security plan procedure for Heavy Goods Vehicles parking areas: An application to the Lazio Region (Italy). Transp. Res. Part E Logist. Transp. Rev. 2014, 65, 35-49. [CrossRef]

3. Coleman, J.A.; Trentacoste, M.F. Model Development For National Assessment of Commercial Vehicle Parking; FHWA-RD-01-159; Turner-Fairbank Highway Research Center: McLean, VA, USA, 2002.

4. Nourinejad, M.; Wenneman, A.; Habib, K.N.; Roorda, M.J. Truck parking in urban areas: Application of choice modelling within traffic microsimulation. Transp. Res. Part A Policy Pract. 2014, 64, 54-64. [CrossRef]

5. Sugumaran, R.; Degroote, J. Spatial Decision Support Systems: Principles and Practices; CRC Press: Boca Raton, FL, USA, 2010. 
6. Jelokhani-Niaraki, M.; Malczewski, J. A group multicriteria spatial decision support system for parking site selection problem: A case study. Land Use Policy 2015, 42, 492-508. [CrossRef]

7. Neisani Samani, Z.; Karimi, M.; Alesheikh, A. A Novel Approach to Site Selection: Collaborative Multi-Criteria Decision Making through Geo-Social Network (Case Study: Public Parking). ISPRS Int. J. Geo-Inf. 2018, 7, 82. [CrossRef]

8. Ferretti, V. From stakeholders analysis to cognitive mapping and Multi-Attribute Value Theory: An integrated approach for policy support. Eur. J. Oper. Res. 2016, 253, 524-541. [CrossRef]

9. Ferretti, V.; Pluchinotta, I.; Tsoukiás, A. Studying the generation of alternatives in public policy making processes. Eur. J. Oper. Res. 2019, 273, 353-363. [CrossRef]

10. Office for National Statistics. 2011 Census. Available online: https://www.ons.gov.uk/census/2011census (accessed on 13 August 2019).

11. Department of Transport. Department of Transport Traffic Counts. Available online: https://www.dft.gov. uk/traffic-counts /cp.php?la=Bradford (accessed on 13 August 2019).

12. Hosseini, S.; Moharerhaye Esfahani, A. Obnoxious Facility Location. In Facility Location; Zanjirani Farahani, R., Hekmatfar, M., Eds.; Contributions to Management Science; Physica: Heidelberg, Germany, 2009.

13. Densham, P.J. Spatial Decision Support Systems. In Geographical Information Systems: Principles and Applications; Maguire, D.J., Goodchild, M.F., Rhind, D.W., Eds.; Longman Scientific \& Technical: Harlow, UK, 1993.

14. Saaty, T.L. Axiomatic Foundation of the Analytic Hierarchy Process. Manag. Sci. 1986, 32, 841-855. [CrossRef]

15. Saaty, T.L. Decision Making With Dependence and Feedback: The Analytic Network Process; RWS Publication: Pittsburgh, PA, USA, 1996.

16. Dente, B. Understanding Policy Decisions; PoliMI SpringerBriefs; Springer: Cham, Switzerland; Heidelberg, Germany, 2014; pp. 1-27.

17. Saaty, T.L. Theory and Applications of the Analytic Network Process: Decision Making With Benefits, Opportunities, Costs, and Risks; RWS Publications: Pittsburgh, PA, USA, 2005.

18. Perron, O. Zur theorie der matrices. Math. Ann. 1907, 64, 248-263. [CrossRef]

(C) 2019 by the authors. Licensee MDPI, Basel, Switzerland. This article is an open access article distributed under the terms and conditions of the Creative Commons Attribution (CC BY) license (http://creativecommons.org/licenses/by/4.0/). 